\title{
Yogic breathing in hypobaric environment: breathing exercising and its effect on hypobaric hypoxemia and heart rate at 3,650-m elevation
}

\author{
Vera Spatenkova ${ }^{1,23, *}$, Roman Bednar ${ }^{4}$, Gabriela Oravcova ${ }^{5}$, Anna Melichova ${ }^{6}$, Eduard Kuriscak ${ }^{3}$ \\ ${ }^{1}$ Faculty of Health Studies, Technical University of Liberec, Liberec, Czech Republic \\ ${ }^{2}$ Neurointensive Care Unit, Neurocenter, Regional Hospital, Liberec, Czech Republic \\ ${ }^{3}$ Institute of Physiology, First Medical Faculty, Charles University in Prague, Praha, Czech Republic \\ ${ }^{4}$ Department of Physiotherapy Balneology and Medical Rehabilitation, University Hospital with Polyclinic of F. D. Roosevelt, Banska Bystrica, Slovakia Republic \\ ${ }^{5}$ Clinic of Pneumology and Phtiseology, Martin University Hospital, Martin, Slovakia Republic \\ ${ }^{6}$ Faculty of Health Care, Banska Bystrica, Slovak Medical University, Banska Bystrica, Slovakia Republic
}

High altitude sojourn is a risk factor for hypobaric hypoxemia and subsequent altitude sickness. The aim of this study was to analyze the effect of new type of yogic breathing — Maheshwarananda's new Modified Bhujangini Pranayama performed by active yoga practitionerson the arterial haemoglobin saturation of oxygen (measured by the pulse oximetry - $\mathrm{SpO}_{2}$ ) and the heart rate compared to normal spontaneous resting breathing. A pilot prospective study was conducted in the Himalayas at an altitude of $3,650 \mathrm{~m}$. We monitored $\mathrm{SpO}_{2}$ and pulse rate in 34 experienced yoga practitioners. Within the 3 measurement days at the altitude of $3,650 \mathrm{~m}$, the mean value of $\mathrm{SpO}_{2}$ increased from $89.11 \pm$
4.78 to $93.26 \pm 4.44(P<0.001)$ after the yogic breathing exercise. No significant changes were observed in pulse rate $(P<0.230)$ measured before and after yogic breathing. The new Yogic breathing - Maheshwarananda's Modified Bhujangini Pranayama - is increasing the arterial haemoglobin saturation compared to normal resting spontaneous breathing. The heart rate was not affected by this type of yogic breathing.

Keywords: Hypoxia, Yogic breathing, Bhujangini Pranayama, Hemoglobin saturation, Altitude sickness

\section{INTRODUCTION}

The high altitudes of the Himalayas are considered to be very demanding and exhausting environment with considerable risk of hypobaric hypoxemia and subsequent altitude sickness. Over the first hours and days of sojourn, the organism commences compensatory processes to mitigate the effects of hypoxia. Ventilation and heart rate are increased, however with no significant increase in stroke volumes (Naeije, 2010). This is a common finding demonstrating reduced plasma volume few days after the acclimatization (Hannon et al., 1969; Siebenmann et al., 2013), being explained by an increased oxygen-carrying capacity of blood unite volume caused by hemoconcentration, hypovolemia, and decreased dia- stolic filling lowering the stroke volume. During prolonged sojourn at higher altitudes, an enhanced erythropoesis results in higher production of haemoglobin mass and allows for a partial or full replenishment of circulatory blood volume and improvement of diastolic functions of the heart (Bärtsch and Saltin, 2008). The effects of hypobaric hypoxia and corresponding physiological adjustments depend naturally not only on the actual elevation but also on the level of physical activity (Deweber and Scorza, 2010; Khodaee et al., 2016; Wolfel et al., 1991) and rate of ascent (Hainsworth et al., 2007).

Increased ventilation at higher altitudes causes a respiratory alkalosis that is counter-balanced within 1 day at altitudes around 2,200 $\mathrm{m}$ above sea level, while remains incomplete at an altitude
${ }^{*}$ Corresponding author: Vera Spatenkova (D) https://orcid.org/0000-0003-4989-0967 Faculty of Health Studies, Technical University of Liberec, Studentská 1402/2, 46117 Liberec 1, Czech Republic Email: vera.spatenkova@nemlib.cz Received: June 2, 2021 / Accepted: July 1, 2021
This is an Open Access article distributed under the terms of the Creative Commons Attribution Non-Commercial License (https://creativecommons.org/licenses/by-nc/4.0/) which permits unrestricted non-commercial use, distribution, and reproduction in any medium, provided the original work is properly cited. 
around 4,100 m (Dempsey et al., 1972; Lundby et al., 2004). Full and partial-to-full compensation of respiratory alkalosis is accomplished by increased renal bicarbonate excretion that is resulting in a reduced buffer capacity of the blood plasma (Böning et al., 2008). Hypoxia is a well-known potent condition which activates a sympathetic nervous system response via known (chemoreceptor and baroreceptor reactions, pulmonary hypertension due to vasoconstriction in lungs; Hainsworth et al., 2007), and also via less known, or yet undiscovered mechanisms (deployed likely in more extreme and less explored conditions). Generally speaking, the endogenous homeostatic systems carry out automatically the inherent sequences of compensatory reactions, yet performing adaptive resetting of parameters being regulated, doing so according to an actual body state and anticipatory systems working based on experience and memory. In addition to that, there is a not very well explored space for voluntary control that can partially modify the autonomous reactions, including the alteration of breathing pattern, and this is what we like to address here within the scope of regulatory physiology, yogic exercise, and yogic breathing.

Bernardi et al. (2007) have shown that a yoga-derived respiratory technique or even simple regularization of breathing at a slow rate could increase the $\mathrm{HbO}_{2}$ saturation and exercise capacity in chronic heart failure. Analogous respiratory patterns in normal individuals could have a similar effect during exposure to hypobaric hypoxia, conditions encountered during a high-altitude sojourn. They mentioned that practitioners of yoga successfully adapt to high altitudes owing to the ventilation pattern controlled voluntarily by yoga supervision, with a smaller decline in oxygen saturation (Bernardi et al., 2001) compared to normal spontaneous breading. Miles (1964) observed that practicing Pranayama (a type of yoga breathing exercise) could have a positive role in physiological adjustments to high altitudes and improve the delivery of oxygen to compensate an acute phase of hypoxia. It was also found that a lower frequency of breathing under yogic exercising reduces the sensitivity of hypoxic and hypercapnic chemo-reflex (Spicuzza et al., 2000), what along with the breathing pattern is discussed and studied further by Bernardi et al. (2007) focusing on Caucasian yoga trainees and Himalayan monks and Sherpas (compared with a respective intra-ethnic control group). Bhujangini Pranayama studied in this work is a type of yogic breathing with slow and deep inspirations through both nostrils and slow expirations through the mouth. Around $90 \%$ of Himalayan Sherpas and monks exhibit the breathing pattern that is very similar to the "yogic breathing" with slow breathing rates. Himashree et al. (2016) published a study on 200 soldiers at an altitude of
3,445 m, demonstrating that practitioners of yoga showed improvement in relevant biochemical parameters at this altitude, compared to the control group. It is long been suggested that comprehensive yoga exercises, via consciously and voluntarily controlled respiration pattern, can induce hypometabolic status and allow trainees to tolerate hypoxia more easily (Wallace et al., 1971), indicating that this can be in some respects a good strategy to cope with high altitude hypoxia (Bernardi et al., 2007).

The aim of this pilot study was to find out whether the new yogic breathing — the Maheshwarananda's Modified Bhujangini Pranayama-increases the saturation of oxygen in the arterial blood (measured by $\mathrm{SpO}_{2}$ sensor) at an altitude of 3,650 $\mathrm{m}$ in active yoga practitioners. Another goal of this study was to see the effect of this yogic breathing on the heart rate.

\section{MATERIALS AND METHODS}

\section{Participants}

An international pilot prospective one-group study was conducted in September 2018 at Nanda Devi National Park in the Alakhpuri Mountains in the Indian Himalayas at an altitude of $3,650 \mathrm{~m}$. The study included 34 subjects with mean age $50.62 \pm$ 13.12 years (16-74 years old), 10 men (29.41\%) with mean age $44.40 \pm 13.66$, and 24 women (70.59\%) with mean age $53.21 \pm$ 12.25. Most individuals were from Slovakia (24, 70.59\%), fewer from Czechia $(9,26.47 \%)$, and one participant (2.94\%) was from Croatia. These 34 subjects were included in one study group.

The study was approved by Ethics Committees No. 8918 of the University Hospital with the Polyclinic of F. D. Roosevelt Banska Bystrica, Slovakia, which had approved the clinical use of our new, easier modification of Maheshwarananda's Bhujangini Pranayama.

Travel schedule: (a) 2 days at an altitude of $372 \mathrm{~m}$ in Rishikesh (time T0) upon arrival at New Delhi airport; (b) 2 days at an altitude of 3,300 $\mathrm{m}$ in Badrinath (time T1) - this altitude was reached during one day via bus transportation; (c) 6 days spent in camp at an altitude of 3,650 $\mathrm{m}$ ( 3 of these 6 days were measurements days: 3 rd day $=\mathrm{T} 2$, 4th day $=\mathrm{T} 3$, and 5 th day $=\mathrm{T} 4$, on 6th day, participants were leaving the camp).

Inclusion criteria were defined as (a) yoga exercisers with at least 5-year experience in the system Yoga in Daily Life (a detailed description of this system and further references related to it could be found in Pukšić et al. 2021); (b) daily practice of a modified Bhujangini Pranayama breathing exercise in the camp for 3 consecutive days; (c) no chronic disease; (d) nonsmokers; (e) lactovegetarians; (f) teetotal; (g) treatments - medications during the study: 
all medications finished on T4, 2 subjects took two 125-mg doses of Acetazolamide per day from T1 to T4 and 5 subjects took two 250-mg doses of Ginkgo biloba per day from T0 to T4, while $400 \mathrm{mg}$ of Ibuprofen was taken sporadically due to the headaches from T1 to T4, no other drugs were taken by the subjects. Taking into consideration described inclusion criteria, especially the longterm yoga experience of tested subjects, we believe, the study group we worked with can be considered quite homogenous in terms of giving reproducible data regarding the studied breathing pattern.

We measured two parameters: (a) $\mathrm{SpO}_{2}$ and (b) pulse heart rate (also measured with the pulse oximeter). The parameters were measured before and after Modified Bhujangini Pranayama breathing exercise on the right forefinger, one subject per time, by two physicians, using the finger pulse oximeter SPO25 (type SPO25, model no. SPO18, company name: Hans Dinslage GmbH, Uttenweller, Germany; one device was used for all measurements, two doctors worked simultaneously giving instructions to the subject, watching the procedure, the position of the body and proper placement of the oximeter, its reading, etc.). The $\mathrm{SpO}_{2}$ device we worked with is the sensor with declared "accuracy" $\pm 2 \%$ for saturation range $70 \%-100 \%$ and with $\pm 2 \%$ beats/minute for the heart rate within $30 \mathrm{bpm}-250 \mathrm{bpm}$ range. The measurements were made at 3 different altitudes: (a) $372 \mathrm{~m}$ (time T0); (b) 3,300 m (time T1); (c) 3,650 m (time $\mathrm{T} 2, \mathrm{~T} 3$ and $\mathrm{T} 4$ ).

We tested Maheshwarananda's new Modified Bhujangini Pranayama type of breathing, which differs from a standard Bhujangini Pranayama type of breathing in a way how and onto which part of the respiratory system is the mental concentration of the subject focused. The standard Pranayama breathing exercise aims the attention on perceiving the feelings in the throat, whereas in the modified version the emphasis is put on perceiving an air volume and its flow in the lungs and chest region. The pattern of breathing in both yogic breathing exercises is very similar, consisting of slow, deep inspiration through both nostrils and a slow, prolonged expiration through the mouth (for additional information and further references see Pukšić et al. 2021). Expiration is longer than inspiration (2-3 times), the duration of expiration varies according to the individual tolerance to prolonged expirations. The yoga breathing and oximetry measurements took place in a yoga sitting position, either with crossed legs or sitting on heels (type of sitting was voluntary), but the back was straight, upper limbs were bent at the elbow joints, and subjects laid on the knees with eyes closed. Yoga breathing exercises were conducted outdoors and everybody was warmly dressed and felt thermo-neutral.

\section{Study protocol}

The yoga breathing examination protocol (Protocol) was conducted early morning and also at lunchtime. It was made up of the following phases: 1 . normal breathing lasting 3 min: upon arrival of each tested subject at the measurement site (reached by short nonstrenuous walking), each individual was instructed to stay approximately $3 \mathrm{~min}$ at rest, in the sitting position, and breath spontaneously (normal, quiet breathing); 2 . immediately after the first phase, the $\mathrm{SpO}_{2}$ and pulse heart rate was measured (reading lasted approximately $1 \mathrm{~min}$ ) with pulse oximeter placed on the right index finger. During this phase subjects were sitting with back in a straight position and continuing normal, quiet resting breathing. These measurements were taken as reference breathing pattern - the control breathing pattern in our study; 3. then each individual started the Modified Bhujangini Pranayama breathing, completed 20 breathing cycles (this phase lasted 2-3 min, so the respiratory frequency ranged from 6 to 10 breaths per min) and indicated the end of this phase by raising the index finger; 4 . then again, the reading of $\mathrm{SpO}_{2}$ and pulse heart rate started immediately afterwards and lasted approximately $1 \mathrm{~min}$.

\section{Statistical analysis}

Data analysis was performed in MS Excel 2013 with descriptive parameters processed in XLSTAT. We used the Shapiro-Wilk test and Jarque-Bera test to verify the normality of the data. The statistical significance of measured value differences was verified using pair tests: $t$-test: paired two samples for means and Wilcoxon signed-rank test. Multiple pairwise comparisons using Nemenyi's procedure (after Friedman test/two-tailed test) were used for $\mathrm{SpO}_{2}$ at the altitude of 3,650 $\mathrm{m}$ after Pranayama between days. Oneway repeated-measures analyis of variance with subsequent Tukey was used in pulse at 3,650 m after Pranayama between days (analysis of the differences between the categories with a confidence interval of $99 \%$ ).

\section{RESULTS}

Out of the 34 yoga practitioners, 5 were excluded from the study due to an acute upper and lower respiratory tract inflammation and camp virus at 3,650 $\mathrm{m}$. Twenty-nine remaining subjects (mean age, $49.76 \pm 13.11$ years), made up by 20 women (mean age, $52.40 \pm$

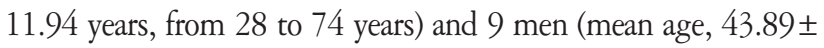
14.38 years, from 16 to 67 years) underwent the measurement Protocol (see MATERIALS AND METHODS section). The initial measured parameters during the normal resting breathing 
(phase 2 of the Protocol - before yoga breathing exercise) at individual altitudes are shown in Table 1 . Table 2 shows analyzed pa- rameters before (phase 2 of the Protocol, normal resting breathing) and after the yogic breathing (phase 4 of the Protocol) and

Table 1. The initial (phase 2 of the protocol - before Pranayama breathing exercise) saturation of oxygen $\left(\% \mathrm{SpO}_{2}\right)$ and pulse rate at individual altitude elevations (T0 - $372 \mathrm{~m}, \mathrm{~N}=34 ; \mathrm{T} 1$ - 3,300 m, N=23; 3 days at 3,650 m: T2=3rd day, $\mathrm{N}=29 ; \mathrm{T} 3=4$ th day, $\mathrm{N}=27 ; \mathrm{T} 4=5$ th day, $\mathrm{N}=29$ )

\begin{tabular}{|c|c|c|c|c|c|c|c|c|c|c|}
\hline \multirow[b]{2}{*}{ Parameter } & \multicolumn{2}{|c|}{ TO - 372 m } & \multicolumn{2}{|c|}{$\mathrm{T} 1$ - 3,300 m } & \multicolumn{2}{|c|}{$\mathrm{T} 2-3,650 \mathrm{~m}$} & \multicolumn{2}{|c|}{ T3 - 3,650 m } & \multicolumn{2}{|c|}{$\mathrm{T} 4$ - 3,650 m } \\
\hline & $\begin{array}{c}\mathrm{SpO}_{2} \\
\left(\% \mathrm{SpO}_{2}\right)\end{array}$ & $\begin{array}{c}\text { Pulse } \\
\text { (beats/min) }\end{array}$ & $\begin{array}{c}\mathrm{SpO}_{2} \\
\left(\% \mathrm{SpO}_{2}\right)\end{array}$ & $\begin{array}{c}\text { Pulse } \\
\text { (beats/min) }\end{array}$ & $\begin{array}{c}\mathrm{SpO}_{2} \\
\left(\% \mathrm{SpO}_{2}\right)\end{array}$ & $\begin{array}{c}\text { Pulse } \\
\text { (beats/min) }\end{array}$ & $\begin{array}{c}\mathrm{SpO}_{2} \\
\left(\% \mathrm{SpO}_{2}\right)\end{array}$ & $\begin{array}{c}\text { Pulse } \\
\text { (beats/min) }\end{array}$ & $\begin{array}{c}\mathrm{SpO}_{2} \\
\left(\% \mathrm{SpO}_{2}\right)\end{array}$ & $\begin{array}{c}\text { Pulse } \\
\text { (beats/min) }\end{array}$ \\
\hline Total group & $96.44 \pm 1.64$ & $75.82 \pm 10.16$ & $88.57 \pm 2.98$ & $86.39 \pm 14.79$ & $87.93 \pm 4.93$ & $84.07 \pm 16.06$ & $89.00 \pm 4.87$ & $81.74 \pm 17.49$ & $90.38 \pm 4.38$ & $77.34 \pm 16.23$ \\
\hline Male & $97.00 \pm 1.15$ & $69.00 \pm 12.36$ & $88.22 \pm 4.15$ & $81.78 \pm 17.25$ & $87.33 \pm 4.09$ & $80.11 \pm 19.64$ & $89.38 \pm 2.88$ & $66.63 \pm 14.28$ & $91.00 \pm 3.24$ & $67.56 \pm 16.52$ \\
\hline Female & $96.21 \pm 1.77$ & $78.67 \pm 7.73$ & $88.79 \pm 2.08$ & $89.36 \pm 12.76$ & $88.20 \pm 5.35$ & $85.85 \pm 14.39$ & $88.84 \pm 5.56$ & $88.11 \pm 14.77$ & $90.10 \pm 4.85$ & $81.75 \pm 14.40$ \\
\hline Age $<45$ & $97.18 \pm 0.87$ & $74.46 \pm 11.34$ & $90.27 \pm 1.90$ & $87.64 \pm 14.91$ & $87.90 \pm 3.38$ & $84.70 \pm 15.39$ & $90.89 \pm 2.76$ & $81.33 \pm 18.32$ & $91.10 \pm 2.64$ & $75.30 \pm 18.98$ \\
\hline Age 45-60 & $96.36 \pm 1.91$ & $76.29 \pm 11.30$ & $87.20 \pm 3.23$ & $85.00 \pm 16.35$ & $89.92 \pm 3.02$ & $87.42 \pm 19.41$ & $89.92 \pm 2.23$ & $81.73 \pm 16.89$ & $91.33 \pm 2.90$ & $78.00 \pm 16.19$ \\
\hline Age $>60$ & $95.67 \pm 1.66$ & $76.78 \pm 7.33$ & $88.00 \pm 2.94$ & $88.00 \pm 7.17$ & $84.57 \pm 8.24$ & $77.43 \pm 9.14$ & $85.29 \pm 7.85$ & $82.29 \pm 20.05$ & $87.71 \pm 7.28$ & $79.14 \pm 14.02$ \\
\hline
\end{tabular}

Values are presented as mean \pm standard deviation.

$\mathrm{T} 2=3$ rd day, $\mathrm{T} 3=4$ th day, $\mathrm{T} 4=5$ th day .

Table 2. Changes in oxygen saturation $\left(\mathrm{SpO}_{2}\right)$ and pulse rate after Pranayama breathing exercise for 3 days at $3,650 \mathrm{~m}$

\begin{tabular}{|c|c|c|c|c|c|c|c|}
\hline \multirow{2}{*}{ Parameter } & \multirow{2}{*}{ No. } & \multicolumn{2}{|c|}{ Oxygen saturation ( $\left.\% \mathrm{SpO}_{2}\right)$} & \multirow{2}{*}{$P$-value } & \multicolumn{2}{|c|}{ Pulse rate (beats/min) } & \multirow{2}{*}{$P$-value } \\
\hline & & Before Pranayama & After Pranayama & & Before Pranayama & After Pranayama & \\
\hline \multicolumn{8}{|c|}{ 3,650 m T2 (before eating) } \\
\hline Total group & 29 & $87.93 \pm 4.93$ & $93.21 \pm 3.95$ & $<0.001$ & $84.07 \pm 16.06$ & $82.59 \pm 16.00$ & 0.158 \\
\hline Male & 9 & $87.33 \pm 4.09$ & $92.56 \pm 3.78$ & 0.005 & $80.11 \pm 19.64$ & $80.22 \pm 18.49$ & 0.479 \\
\hline Female & 20 & $88.20 \pm 5.35$ & $93.50 \pm 4.09$ & $<0.001$ & $85.85 \pm 14.39$ & $83.65 \pm 15.14$ & 0.131 \\
\hline Age $<45$ yr & 10 & $87.90 \pm 3.38$ & $92.90 \pm 4.36$ & 0.006 & $84.70 \pm 15.39$ & $82.90 \pm 16.76$ & 0.309 \\
\hline Age $45-60 \mathrm{yr}$ & 12 & $89.92 \pm 2.02$ & $94.25 \pm 3.19$ & $<0.001$ & $87.42 \pm 19.41$ & $83.75 \pm 18.69$ & 0.007 \\
\hline Age $>60$ yr & 7 & $84.57 \pm 8.24$ & $91.86 \pm 4.60$ & 0.017 & $77.43 \pm 9.14$ & $80.14 \pm 1.10$ & 0.141 \\
\hline \multicolumn{8}{|c|}{ 3,650 m T3 (after eating) } \\
\hline Total group & 27 & $89.00 \pm 4.87$ & $92.19 \pm 5.28$ & $<0.001$ & $81.74 \pm 17.49$ & $82.33 \pm 15.35$ & 0.367 \\
\hline Male & 8 & $89.38 \pm 2.88$ & $92.13 \pm 4.52$ & 0.089 & $66.63 \pm 14.28$ & $73.63 \pm 13.52$ & 0.048 \\
\hline Female & 19 & $88.84 \pm 5.56$ & $92.21 \pm 5.69$ & 0.001 & $88.11 \pm 14.77$ & $86.00 \pm 14.88$ & 0.103 \\
\hline Age $<45$ yr & 9 & $90.89 \pm 2.76$ & $93.89 \pm 2.62$ & 0.007 & $81.33 \pm 18.32$ & $83.67 \pm 17.31$ & 0.254 \\
\hline Age $45-60 \mathrm{yr}$ & 11 & $89.82 \pm 2.23$ & $91.45 \pm 5.59$ & 0.125 & $81.73 \pm 16.89$ & $81.55 \pm 14.03$ & 0.472 \\
\hline Age $>60 \mathrm{yr}$ & 7 & $85.29 \pm 7.85$ & $91.14 \pm 7.29$ & 0.007 & $82.29 \pm 20.05$ & $81.86 \pm 17.00$ & 0.455 \\
\hline \multicolumn{8}{|c|}{ 3,650 m T4 (before eating) } \\
\hline Total group & 29 & $90.38 \pm 4.38$ & $94.31 \pm 3.91$ & $<0.001$ & $77.34 \pm 16.23$ & $80.52 \pm 17.85$ & 0.068 \\
\hline Male & 9 & $91.00 \pm 3.24$ & $95.22 \pm 2.44$ & 0.004 & $67,56 \pm 16.52$ & $74.44 \pm 16.53$ & 0.051 \\
\hline Female & 20 & $90.10 \pm 4.85$ & $93.90 \pm 4.41$ & $<0.001$ & $81.75 \pm 14.40$ & $83.25 \pm 18.15$ & 0.275 \\
\hline Age $<45$ yr & 10 & $91.10 \pm 2.64$ & $95.80 \pm 2.94$ & 0.002 & $75.30 \pm 18.98$ & $82.10 \pm 19.17$ & 0.056 \\
\hline Age $45-60 \mathrm{yr}$ & 12 & $91.33 \pm 2.90$ & $94.17 \pm 3.13$ & 0.006 & $78.00 \pm 16.19$ & $80.83 \pm 20.93$ & 0.180 \\
\hline Age $>60$ yr & 7 & $87.71 \pm 7.25$ & $92.43 \pm 5.71$ & 0.017 & $79.14 \pm 14.02$ & $77.74 \pm 10.94$ & 0.369 \\
\hline \multicolumn{8}{|l|}{3,650 m T2-4 } \\
\hline Total group & 85 & $89.11 \pm 4.78$ & $93.26 \pm 4.44$ & $<0.001$ & $81.04 \pm 16.63$ & $81.80 \pm 16.29$ & 0.230 \\
\hline Male & 26 & $89.23 \pm 3.67$ & $93.35 \pm 3.76$ & $<0.001$ & $72.17 \pm 18.02$ & $75.87 \pm 16.91$ & 0.026 \\
\hline Female & 59 & $89.05 \pm 5.23$ & $93.22 \pm 4.74$ & $<0.001$ & $85.19 \pm 14.51$ & $84.27 \pm 15.91$ & 0.220 \\
\hline Age $<45$ yr & 29 & $89.93 \pm 3.22$ & $94.21 \pm 3.52$ & $<0.001$ & $80.41 \pm 17.44$ & $82.86 \pm 17.16$ & 0.129 \\
\hline Age $45-60 \mathrm{yr}$ & 35 & $90.37 \pm 2.45$ & $94.34 \pm 4.17$ & $<0.001$ & $82.40 \pm 17.51$ & $82.06 \pm 17.73$ & 0.404 \\
\hline Age $>60$ yr & 21 & $85.86 \pm 7.52$ & $91.81 \pm 5.69$ & $<0.001$ & $79.62 \pm 14.45$ & $79.91 \pm 12.75$ & 0.441 \\
\hline
\end{tabular}

Values are presented as mean \pm standard deviation.

$\mathrm{T} 2=3 \mathrm{rd}$ day, $\mathrm{T} 3=4$ th day, $\mathrm{T} 4=5$ th day. 


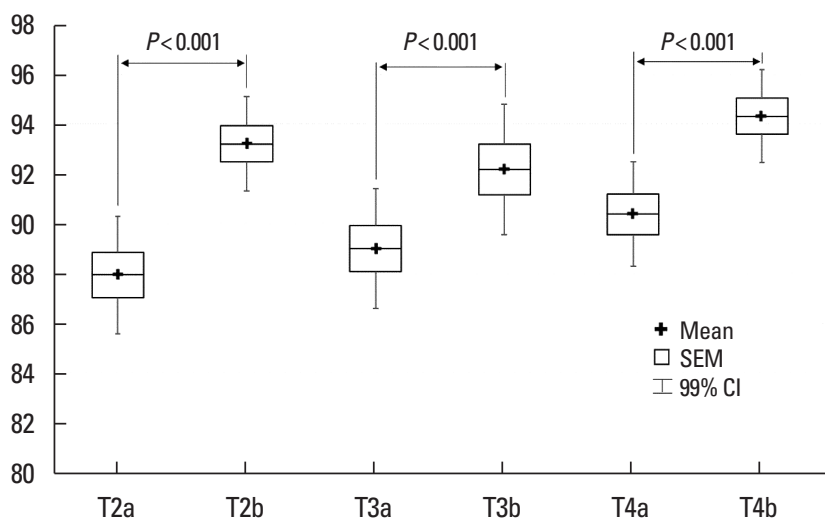

Fig. 1. Changes in oxygen saturation after Pranayama breathing exercise for 3 days at 3,650 $\mathrm{m}$ ( $a=$ before Pranayama breathing exercise, $b=$ after Pranayama breathing exercise, $\mathrm{T} 2=3 \mathrm{rd}$ day, $\mathrm{T} 3=4$ th day, $\mathrm{T} 4=5$ th day). SEM, standard error of mean; $\mathrm{Cl}$, confidence interval.

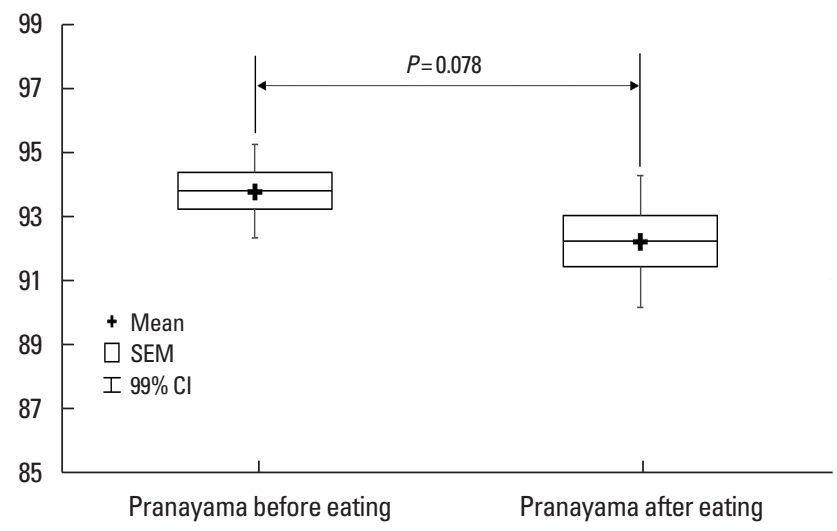

Fig. 2. Changes in oxygen saturation after Pranayama breathing exercise before and after a meal at 3,650 m. SEM, standard error of mean; $\mathrm{Cl}$, confidence interval.

Table 3. Changes in oxygen saturation $\left(\mathrm{SpO}_{2}\right)$ and pulse rate after Pranayama breathing exercise between individual days at 3,650 $\mathrm{m}$

\begin{tabular}{|c|c|c|c|c|c|c|}
\hline \multirow{2}{*}{ Parameter } & \multicolumn{2}{|c|}{ Oxygen saturation (\% $\left.\mathrm{SpO}_{2}\right)$} & \multirow{2}{*}{$P$-value } & \multicolumn{2}{|c|}{ Pulse (beats/min) } & \multirow{2}{*}{$P$-value } \\
\hline & Sample 1 & Sample 2 & & Sample 1 & Sample 2 & \\
\hline T2 total vs. T3 total & $92.96 \pm 3.99$ & $92.19 \pm 5.28$ & 0.8495 & $83.85 \pm 15.34$ & $82.33 \pm 15.35$ & 0.9298 \\
\hline T2 total vs. T4 total & $92.96 \pm 3.99$ & $94.52 \pm 3.92$ & 0.1822 & $83.85 \pm 15.34$ & $82.63 \pm 16.50$ & 0.9572 \\
\hline T3 total vs. T4 total & $92.19 \pm 5.28$ & $94.52 \pm 3.92$ & 0.0558 & $82.33 \pm 15.35$ & $82.63 \pm 16.50$ & 0.9974 \\
\hline
\end{tabular}

Values are presented as mean \pm standard deviation.

$\mathrm{T} 2=$ 3th day, $\mathrm{T} 3=4$ th day, $\mathrm{T} 4=5$ th day.

demonstrates a statistically significant increase of $\mathrm{SpO}_{2}$ after the yoga breathing exercise. At day T2, the whole group's $\mathrm{SpO}_{2}$ average increased from 87.93 to 93.21 , at T3 from 89.00 to 92.19 , and at T4 from 90.38 to 94.31 (averaging over T2, T3, and T4 measurements resulted in an overall increase of $\mathrm{SpO}_{2}$ from 89.11 \pm 4.78 to $93.26 \pm 4.44, P<0.001$ ). Similar results were seen within the distinct age and gender subgroups (see Table 2), indicating that the $\mathrm{SpO}_{2}$ in various age and gender groups changed in the same way due to the yogic breathing, indicating the tested age range or gender did not interfere significantly with measured values of $\mathrm{SpO}_{2}$, assuming all participants mastered this type of yoga breathing relatively equally.

The initial $\mathrm{SpO}_{2}$ values (normal resting breathing - phase 2 of the Protocol) at various altitudes are shown in Table 1. Initial values of $\mathrm{SpO}_{2}$ during the three subsequent days (T2-4, see also Fig. 1) demonstrate a trend of progressive increase of $\mathrm{SpO}_{2}$ at $3,650 \mathrm{~m}$, and may indicate an early phase of acclimatization. Statistically nonsignificant changes were observed in heart rate before (phase 2) and after the yoga breathing exercise (phase 4 of the Protocol) what indicates the tested type of voluntary yogic breathing did not affect the heart rate noticeably.
Table 3 shows the $\mathrm{SpO}_{2}$ as well as the pulse rates after the yogic breathing over the consecutive days T2-3, which indicate a lack of any significant trend. When testing the effect of eating, we found it also did not have any statistically significant association with $\mathrm{SpO}_{2}$ changes induced by the yogic breathing exercise (see Fig. 2).

\section{DISCUSSION}

The regions of high-altitude elevation represent a very demanding area, exhausting quickly physical reserves of the body and posing a significant risk of hypobaric hypoxemia, tissue hypoxia, and subsequent altitude sickness. Any physical activity under the conditions of hypobaric hypoxia becomes more strenuous and further challenges the acute phase of physiological responses, the acclimatization phase, and also the late adaptation mechanisms of our body at high altitudes (Deweber and Scorza, 2010; Khodaee et al., 2016; Wolfel et al., 1991). Modulation of autonomous homeostatic mechanisms reducing the effects of hypoxia is one of the possible strategies that can adjust the accommodation to these demanding conditions, and as some studies indicate (Bernardi et al., 
2001, 2007; Miles, 1964; Spicuzza et al., 2000), a voluntary alteration of spontaneous breathing pattern by practicing yoga breathing exercises could be a very interesting approach. Our study is the first of its kind that is studying the effect of new yogic breathing exercise - Maheshwarananda's Modified Bhujangini Pranaya$\mathrm{ma}$ - on the arterial $\mathrm{HbO}_{2}$ saturation levels (measured by $\mathrm{SpO}_{2}$ sensor) at higher altitudes. The essence of this new breathing method resides in the conscious focusing of subject's concentration on perceiving the breathing in the lungs and chest region instead of perceiving it in the throat. According to our study's participants, and to our own experience, this new type of yogic breathing exercise is considered less demanding and more practicable or feasible compared to the classical Pranayama style of breathing exercise. To ensure the proper practicing of this breathing exercise, enhancing thus the reproducibility of tested breathing pattern, we worked with yoga practitioners who had been practicing the system Yoga in Daily Life for at least five years. The second reason we worked with experienced yoga practitioners was the requirement of the correct sitting position, which is central in the Pranayama system. All subjects practiced this position with an upright spine and corresponding pelvis position that is inherent in a yoga practice.

A statistically significant increase in blood oxygenation was observed after series of 20 breaths of Modified Bhujangini Pranayama breathing exercise, demonstrated by an elevation of $\mathrm{SpO}_{2}$ (increased from 89.11 to 93.26, $P<0.001$, averaged over 3 days T24 , see Table 2). We can interpret these results as showing that this type of breathing can transiently increase the blood oxygenation which would prevail minutes after this type of yogic breathing. Depending on the frequency of practicing, we can assume that the studied Bhujangini Pranayama breathing pattern can help individuals to assist physiological adjustments to higher altitudes if practiced adequately enough during the day. It is reasonable to assume that similar consciously controlled breathing patterns, increasing the alveolar ventilation over values found in spontaneous breathing at similar altitudes, will very probably have comparable effects on blood oxygenation (this assumption, however, was not tested by our Protocol). We can speculate why physiological mechanisms do not cause us to react to higher altitudes similarly - by adjusting the respiratory patterns alike the tested yogic breathing and achieving thus higher arterial saturation levels? We can ponder that our regulatory circuits work with a limited amount of information, being received mainly from unconsciously perceived internal sensors, and do not anticipate or process preferentially the information contained in human plans or intentions to stay days or weeks at high altitudes, and on the top of that, doing a strenuous and highly motivated physical exercise. We believe that such body behavior is rationally reasoned - in order to do an instant, reliable and targeted homeostatic interventions, autonomous regulation mechanisms must process relevant and real-time information gathered mainly from body chemo- or presso-sensors (Hainsworth et al., 2007). However, in order to incorporate or fuse optimally complex voluntary plans or objectives into involuntary basic autonomous reactions, a conscious involvement interfering with/or overriding autonomous modulations (including a breathing pattern generation) could under specific conditions be very advantageous, as it could be the case regarding voluntary modifications of respiration that are characteristic to yogic breathing exercises. It is interesting that as a time period an individual spends at higher altitudes prolongs, longer-term compensations start to take place (respecting already long-lasting effect of high altitude on the body), carrying out more optimized and specialized adaptations. This could possibly be a strategy evolved by Himalayan Sherpas and monks, exhibiting a different respiratory pattern than low altitude population, being similar in its nature to the "yogic breathing" (Bernardi et al., 2007). Despite hypoxia is considered certainly a strong stressing factor starting stereotypic respiratory compensatory mechanisms, the signals received from various cortical areas (conscious modulation), limbic system (emotions, motivations), and hypothalamus (circadian rhythms, food intake, higher autonomous reactions) represent the substrate that eventually introduces the demanded complex information refining the physiological regulations (Myers, 2017), making them very effective, especially in less standard or extreme conditions, provided individuals are mentally and physically healthy.

We present this study to demonstrate that the voluntary alteration of breathing pattern can be helpful in hypoxic conditions. Yoga, which concentrates on a conscious control of breathing could be considered as safe and proven way to introduce such voluntary supervision. According to Bernardi et al. (2001), adopting slow and deep breathing could cause a diaphragm to move better, resulting in an improved ventilation accompanied with complete mobilization of respiratory muscles, achieved in well-trained yoga practitioners after a long-term exercise. The benefits of yoga-like breathing in hypoxic environments certainly vary from person to person, depending on the instantaneous and long-term tuning of sensitive homeostatic systems (Himashree et al., 2016). Adequate tuning of this system can result in an improvement of oxygenation observed by yoga exercises via indirect modulation of chemoreceptor reactions (Bernardi et al., 2001; Plataki et al., 2013) - it 
has been documented that slow breathing rate can modulate chemo-reflex sensitivity (Spicuzza et al., 2000), decrease the sympathetic activation of acute altitude exposure and change the vagal activity (Telles et al., 2014) - effects that are being regularly communicated by Pranayama practitioners.

In this study, we also evaluated the influence of eating on the $\mathrm{SpO}_{2}$ levels measured after the yogic breathing, and we did not reveal any association with $\mathrm{SpO}_{2}$ values. The mean pulse rate values (measured together with the $\mathrm{SpO}_{2}$ ) at T2-4 were also not changed significantly $(P<0.230)$ due to the effect of yogic breathing, demonstrating that the heart rate was affected minimally by Bhujangini Pranayama breathing in our subjects. This finding is very interesting because the tested yoga breathing was increasing the $\mathrm{SpO}_{2}$ levels yet doing that without apparent activation of a sympathetic nervous system and corresponding increase of oxygen demands of working myocardium. Despite a majority of papers that are focusing on the yogic breathing's effect on the heart rate speak about its decrease due to the yogic exercising (Ankad et al., 2011; Krishna et al., 2014; Pramanik et al., 2010; Raghavendra et al., 2013; explaining that as a consequence of decrease in sympathetic/parasympathetic balance), some of them are also referring about its increase following the yogic breathing exercising (Mason et al., 2013; Mohan et al., 1986). It seems that whether the heart rate will be increased or decreased depends on a particular breathing technique, on a proportion between inspiration and expiration period, an expiratory pressure (e.g., ujjayi type of resistance breathing) or an $\mathrm{O}_{2}$ partial pressure playing important roles in the resulting $\mathrm{SpO}_{2}$ changes (Mason et al., 2013).

As there is a continuous scientific debate focusing on the precision and accuracy of oximetry devices used in various fields of medicine (Lauterbach 2020; Lipnick et al., 2016; Luks and Swenson, 2020), we would like to discuss some of oximetry limitations and their impact on our results. It has to be mentioned that every device reads the true real values with a certain level of precision and accuracy. Medical devices marketed for medical use in the United States are required by Food and Drug Administration to have the root mean square error (RMSQ) less than $3 \%$ at $\mathrm{SaO}_{2}$ levels within the range of 70\%-100\% (ISO 80601-2-61, 2017). RMSQ reflects both the systematic (biased) error as well as the random error. The systematic error, resulting in an offset from the true value affects the measurement in a constant way with repeated measurements and its effect is thus predictable (the offset may however depend on the actual true value of measured quantity). The random error is causing one measurement to differ from the next, is unpredictable, and is causing fluctuation around the true value. One systematic study analysing the precision of few oximeters (Lipnick et al., 2016; focusing on similar-grade sensors we worked with) showed that the systematic error, expressed as "mean bias" (offset from the true value) was $\mathrm{SaO}_{2}$ value-dependent, and in average largest (-4.56\% to $7.53 \%$ ) for $\mathrm{SaO}_{2}$ range of $70 \%-80 \%$. For the $\mathrm{SaO}_{2}$ range between $80 \%-90 \%$, it was from $-2.73 \%$ to $5.44 \%$ and for $90 \%-100 \%$ the offset was $-0.85 \%$ to $1.2 \%$. These findings, however, demonstrate that despite the systematic errors between various sensor types differed considerably, for one piece of device and a given true value of $\mathrm{SaO}_{2}$ being measured, it was rather constant and its real impact on measured values is basically aberration of scaling and shifting of the ideal measuring gauge by some (device dependent) but constant factor (or function) that can be empirically estimated by detailed systematic testing of a given oximeter. However, even if that scaling factor (or function) is not known to the experimenter (or us), it is still meaningful to do the $\mathrm{SpO}_{2}$ measurement without significant depreciation of its accuracy, provided all values are measured with the same piece of device and the hypothesis of equal means between the two groups is being statistically tested (after finite number of repeated measurements the difference between two different real $\mathrm{SpO}_{2}$ values must become apparent, no matter how large is the systematic error, because an ordering of values is not affected by that error).

The random error, on the other hand, would increase the number of samples needed to reject the null hypothesis at the given level of significance. However, that intrinsic random error of the $\mathrm{SpO}_{2}$ sensor is considered to be only one of many kinds of random observational errors (or natural fluctuations) interfering with the true readings of $\mathrm{SaO}_{2}$ values (which may depend on the placement of $\mathrm{SpO}_{2}$ sensor, pulse quality, possible movement of the finger, duration of the measurement, skin pigmentation, etc.; Chan et al., 2013). Common statistical inferential methods we used in our study do process that inherent random error (as well as any other natural fluctuations smearing the true value) already in their statistics, incorporating it in the final $P$-value at which the null hypothesis is tested. Counting with the random error originating from our sensor estimated by its manufacturer to be close to $\pm 2 \%$, being comparable with an average value of some of the $\mathrm{SpO}_{2}$ sensors of our quality class or grade (Lipnick et al., 2016; with random error reaching $3.4 \%$ in average - expressed as $\mathrm{SD}$ of true $\mathrm{SpO}_{2}$ value), and taking into account our $P$-values were smaller than 0.001 in many of our results, we would like to conclude that it is unlikely the random intrinsic error of our $\mathrm{SpO}_{2}$ sensor has affected our statistical inference significantly (the same argumentation should hold, according to our reasoning, for measurement of the heart rate). 
Another issue related to the $\mathrm{SpO}_{2}$ reading by pulse oximeters is time delays between ventilation and $\mathrm{SpO}_{2}$ changes that commonly range, due to the blood perfusion delays into different parts of our body, from $20 \mathrm{sec}$ to $30 \mathrm{sec}$ (if the oximeter is on the earlobe, or forehead), up to $2 \mathrm{~min}$ if the oximeter is on the finger (MacLeod et al., 2005; Severinghaus and Kelleher, 1992). Because in our $\mathrm{SpO}_{2}$ measurements the period of yogic breathing lasted from 2 to $3 \mathrm{~min}$, which was immediately followed by the period of $\mathrm{SpO}_{2}$ measurement (as mentioned in MATERIALS AND METHODS section), we conclude those delays did not affect measured $\mathrm{SpO}_{2}$ values and their correlation with the yogic breathing.

Other limitation we would like to discuss here is that we managed to accomplish only three series of measurements at an altitude of $3,650 \mathrm{~m}$. Secondly, we did not observe the fading or time dynamic of measured breathing effects on $\mathrm{SpO}_{2}$ levels after the cessation of breathing exercise. The acclimatization period was minimal in our yoga practitioners and thus its effect was not studied as well as altitude sickness manifestations were not analyzed in this pilot study. Further studies are planned to extend our results, including not only experienced yoga practitioners but also a broader spectrum of population capable of adopting similar breathing exercising while staying at high altitudes.

\section{CONFLICT OF INTEREST}

No potential conflict of interest relevant to this article was reported.

\section{ACKNOWLEDGMENTS}

The authors received no financial support for this article. All participants were fully informed regarding this breathing method and agreed to participate in the study and gave consent to publication. We thank the translator and native English speaker Henry Morgan for the correction of the English text.

\section{REFERENCES}

Ankad RB, Herur A, Patil S, Shashikala GV, Chinagudi S. Effect of shortterm pranayama and meditation on cardiovascular functions in healthy individuals. Heart Views 2011;12:58-62.

Bärtsch P, Saltin B. General introduction to altitude adaptation and mountain sickness. Scand J Med Sci Sports 2008;18 Suppl 1:1-10.

Bernardi L, Passino C, Spadacini G, Bonfichi M, Arcaini L, Malcovati L, Bandinelli G, Schneider A, Keyl C, Feil P, Greene RE, Bernasconi C.
Reduced hypoxic ventilatory response with preserved blood oxygenation in yoga trainees and Himalayan Buddhist monks at altitude: evidence of a different adaptive strategy? Eur J Appl Physiol 2007;99: 511-518.

Bernardi L, Passino C, Wilmerding V, Dallam GM, Parker DL, Robergs RA, Appenzeller O. Breathing patterns and cardiovascular autonomic modulation during hypoxia induced by simulated altitude. J Hypertens 2001;19:947-958

Böning D, Rojas J, Serrato M, Reyes O, Coy L, Mora M. Extracellular pH defense against lactic acid in untrained and trained altitude residents. Eur J Appl Physiol 2008;103:127-137.

Chan ED, Chan MM, Chan MM. Pulse oximetry: understanding its basic principles facilitates appreciation of its limitations. Respir Med 2013; 107:789-799.

Dempsey JA, Forster HV, Birnbaum ML, Reddan WG, Thoden J, Grover $\mathrm{RF}$, Rankin J. Control of exercise hyperpnea under varying durations of exposure to moderate hypoxia. Respir Physiol 1972;16:213-231.

Deweber K, Scorza K. Return to activity at altitude after high-altitude illness. Sports Health 2010;2:291-300.

Hainsworth R, Drinkhill MJ, Rivera-Chira M. The autonomic nervous system at high altitude. Clin Auton Res 2007;17:13-19.

Hannon JP, Chinn KS, Shields JL. Effects of acute high-altitude exposure on body fluids. Fed Proc 1969;28:1178-1184.

Himashree G, Mohan L, Singh Y. Yoga practice improves physiological and biochemical status at high altitudes: a prospective case-control study. Altern Ther Health Med 2016;22:53-59.

ISO 80601-2-61:2017. Medical electrical equipment-Part 2-61: particular requirements for basic safety and essential performance of pulse oximeter equipment. Geneva (Switzerland): International Organization for Standardization; 2017 [cited 2021 May 18]. Available from: https:// www.iso.org/standard/67963.html.

Khodaee M, Grothe HL, Seyfert JH, VanBaak K. Athletes at high altitude. Sports Health 2016;8:126-132.

Krishna BH, Pal P, G K P, J B, E J, Y S, M G S, G S G. Effect of yoga therapy on heart rate, blood pressure and cardiac autonomic function in heart failure. J Clin Diagn Res 2014; 8:14-16.

Lauterbach CJ, Romano PA, Greisler LA, Brindle RA, Ford KR, Kuennen MR. Accuracy and reliability of commercial wrist-worn pulse oximeter during normobaric hypoxia exposure under resting conditions. Res Q Exerc Sport 2021;92:549-558.

Lipnick MS, Feiner JR, Au P, Bernstein M, Bickler PE. The accuracy of 6 inexpensive pulse oximeters not cleared by the rood and drug administration: the possible global public health implications. Anesth Analg 2016;123:338-345.

Luks AM, Swenson ER. Pulse oximetry for monitoring patients with 
COVID-19 at home. Potential pitfalls and practical guidance. Ann Am Thorac Soc 2020;17:1040-1046.

Lundby C, Calbet JA, van Hall G, Saltin B, Sander M. Pulmonary gas exchange at maximal exercise in Danish lowlanders during $8 \mathrm{wk}$ of acclimatization to 4,100 $\mathrm{m}$ and in high-altitude Aymara natives. Am J Physiol Regul Integr Comp Physiol 2004;287:R1202-R1208.

MacLeod DB, Cortinez LI, Keifer JC, Cameron D, Wright DR, White WD, Moretti EW, Radulescu LR, Somma J. The desaturation response time of finger pulse oximeters during mild hypothermia. Anaesthesia 2005; 60:65-71.

Mason H, Vandoni M, Debarbieri G, Codrons E, Ugargol V, Bernardi L. Cardiovascular and respiratory effect of yogic slow breathing in the yoga beginner: what is the best approach? Evid Based Complement Alternat Med 2013;2013:743504.

Miles Wr. Oxygen consumption during three yoga-type breathing patterns. J Appl Physiol 1964;19:75-82.

Mohan M, Saravanane C, Surange SG, Thombre DP, Chakrabarty AS. Effect of yoga type breathing on heart rate and cardiac axis of normal subjects. Indian J Physiol Pharmacol 1986;30:334-340.

Myers B. Corticolimbic regulation of cardiovascular responses to stress. Physiol Behav 2017;172:49-59.

Naeije R. Physiological adaptation of the cardiovascular system to high altitude. Prog Cardiovasc Dis 2010;52:456-466.

Plataki M, Sands SA, Malhotra A. Clinical consequences of altered chemoreflex control. Respir Physiol Neurobiol 2013;189:354-363.

Pramanik T, Pudasaini B, Prajapati R. Immediate effect of a slow pace breathing exercise Bhramari pranayama on blood pressure and heart rate. Nepal Med Coll J 2010;12:154-157.

Pukšić S, Mitrović J, Čulo MI, Živković M, Orehovec B, Bobek D, Morović-Vergles J. Effects of Yoga in Daily Life program in rheumatoid arthritis: A randomized controlled trial. Complement Ther Med 2021;57:102639.

Raghavendra B, Telles S, Manjunath N, Deepak K, Naveen K, Subramanya $\mathrm{P}$. Voluntary heart rate reduction following yoga using different strategies. Int J Yoga 2013;6:26-30.

Severinghaus JW, Kelleher JF. Recent developments in pulse oximetry. Anesthesiology 1992;76:1018-1038.

Siebenmann C, Hug M, Keiser S, Müller A, van Lieshout J, Rasmussen P, Lundby C. Hypovolemia explains the reduced stroke volume at altitude. Physiol Rep 2013;1:e00094.

Spicuzza L, Gabutti A, Porta C, Montano N, Bernardi L. Yoga and chemoreflex response to hypoxia and hypercapnia. Lancet 2000;356:14951496.

Telles S, Sharma SK, Balkrishna A. Blood pressure and heart rate variability during yoga-based alternate nostril breathing practice and breath awareness. Med Sci Monit Basic Res 2014;20:184-193.

Wallace RK, Benson H, Wilson AF. A wakeful hypometabolic physiologic state. Am J Physiol 1971;221:795-799.

Wolfel EE, Groves BM, Brooks GA, Butterfield GE, Mazzeo RS, Moore LG, Sutton JR, Bender PR, Dahms TE, McCullough RE. Oxygen transport during steady-state submaximal exercise in chronic hypoxia. J Appl Physiol (1985) 1991;70:1129-1136. 